medication on discharge compared to admission. This will be demonstrated by a reduced score on the Anticholinergic Cognitive Burden (ACB) scale on discharge compared to admission. Target: $80 \%$.

Where new medicines with anticholinergic burden are prescribed during admission, there should be evidence that the anticholinergic properties of these medications have been considered prior to prescribing (via documentation in care co-ordination reviews or progress notes). Target: $100 \%$

Method. Electronic records were searched for all discharges from Roker ward between 1/1/2019 - 31/12/2019. For each record the follwing information was recorded: demographics; primary diagnosis; total ACB score on admission; and total ACB score on discharge. For all new medications started with an ACB score of over zero, records were searched to establish whether there was evidence that the anticholinergic properties of these medications had been considered.

Result. 47 patients were identified who were discharged over the time period in question. 30 patients had no difference in ACB score between admission and discharge; 10 patients had a reduction in ACB score and 5 patients had an increase. A total of 9 new medications with ACB scores over zero had been started during all admissions; there were no occasions where there was documented evidence to show that the anticholinergic burden of these medications had been considered.

Conclusion. $27 \%$ of patients had a reduction in their total ACB score during admission; the target was $80 \%$.

The reasons for starting medications with an ACB score of greater than 1 were documented in $0 \%$ of cases; the target was $100 \%$.

As both targets were missed by a significant margin, it was recognised that there were significant areas for improvement. The following plan was therefore implemented:

1. Following discussion with the ward consultant and ward pharmacist, regular prescriber meetings have been set up which involve senior nursing staff, medical staff and pharmacy - anticholinergic burden is calculated for each patient as part of these meetings

2. A re-audit is recommended after 6 months.

Assessing the quality of risk assessment conducted for new psychiatry inpatients

Nikhita Handa

East Lancashire Hospitals NHS Trust

doi: 10.1192/bjo.2021.251

Aims. An audit was conducted to assess if thorough risk assessments had been documented in electronic clinical record notes (ECR) clerking for new patients in two acute mental health wards. Risk assessment is a vital part of admission clerking and when done well it can prevent early incidents and aid the ward nursing team greatly. During induction, junior doctors are advised to document assessed risks when clerking a new patient. A screening of the risks on admission could help determine the levels of observations required to minimise the identified risks whilst the patient awaits their first ward review.

Method. The NHS numbers for the 30 current inpatients across male and female acute psychiatric wards were gathered at the time of the audit (February - March 2020). Admission clerking was analysed for a clear statement of patient risk to self, others or property. Within these categories quantitative results were obtained on how often the risk of self-harm, self-neglect, absconding, vulnerability or aggression was documented. The term 'risk' was used for each patient on their ECR notes to search for risk assessments in all entries other than admission clerking. Result. 12 out of the 30 patients had a junior doctor risk assessment documented in their clerking (40\%). 14 patients had no mention of risk assessment on admission (47\%) and their first formal risk assessment was documented only in their senior ward review. Of the 12 assessments completed in clerking; all assessed self harm/suicide risk and violent risk to others, 1 mentioned risk of absconding, 8 mentioned risk of illicit substance use and 8 mentioned vulnerability. It was unclear if the risks documented were based on current or historic presentation. Junior doctors were anonymously surveyed following this audit and reported they did not feel confident in how to document a risk assessment or whether to document negative findings.

Conclusion. Clear documentation of risk assessment being performed was lacking in over half of junior doctor admission clerkings. When risks were assessed it was mainly violence/self harm risk documented not vulnerability and physical health risks. Based on these findings we have designed more comprehensive teaching on risk assessments and a template for how to complete a risk assessment. We feel the use of a template will ensure all elements of risk are clearly considered even if they are not present currently. This is being reaudited to assess if the changes have impacted the quality of risk assessment conducted.

\section{Audit of the impact of the integrated psychological medicine service (IPMS) on service utilisation}

Sarah Harvey ${ }^{1 \star}$, Joanna Bromley ${ }^{1}$, Miles Edwards ${ }^{2}$, Megan Hooper ${ }^{1}$, Hannah McAndrew ${ }^{3}$ and Joanne Timms ${ }^{1}$

${ }^{1}$ Devon Partnership Trust; ${ }^{2}$ RD\&E Hospital and ${ }^{3}$ Exeter Medical School

${ }^{*}$ Corresponding author.

doi: 10.1192/bjo.2021.252

Aims. An audit to assess the impact of an Integrated Psychological Medicine Service (IPMS) on healthcare utilization pre \& post intervention. We hypothesized that an IPMS approach would reduce healthcare utilization.

Background. The IPMS focusses on integrating biopsychosocial assessments into physical healthcare pathways. It has developed in stages as opportunities presented in different specialities leading to a heterogeneous non-standardised service. The key aim is involvement of mental health practitioners, psychologists \& psychiatrists in complex patients with comorbidity or functional presentations in combination with the specialty MDT. This audit is the first attempt to gather data across all involved specialities and complete a randomised deep dive into cases.

Method. Referrals into IMPS from July 2019 to June 2020 pulled 129 referrals, of which a $10 \%$ randomised sample of 13 patients was selected to analyse. 5 patients had one year of data either side of the duration of the IPMS intervention (excluding 8 patients with incomplete data sets).

We analysed; the duration \& nature of the IPMS intervention, the number, duration \& speciality of inpatient admissions \& short stays, outpatient attendances, non-attendances \& patient cancellations. Psychosocial information was also gathered. One non-randomised patient was analysed as a comparative case illustration.

Result. Randomised patients; patient 78's utilisation remained static, patient 71 post-referral engaged with health psychology \& reduced healthcare utilisation. Patient 7 increased healthcare utilisation post-referral secondary to health complications. Patient 54 
did not attend \& increased healthcare utilisation post-referral. Patient 106 had increased healthcare utilisation post-referral from a new health condition. The randomised sample identified limitations of using healthcare utilisation as an outcome measure when contrasted to the non-randomised case (which significantly reduced healthcare utilisation post-referral).

Conclusion. Correlation only can be inferred from the data due to sample size, limitations \& confounding factors e.g. psycho-social life events, acquired illness. Alternative outcome measurements documented (e.g PHQ9/GAD7) were not reliably recorded across pathways.

The results evidenced that single cases can demonstrate highly desirable effects of a biopsychosocial approach but they can also skew data sets if results are pooled due to the small sample size \& heterogeneous interventions. With some patients an increase in healthcare utilisation was appropriate for an improved clinical outcome. This audit identified that utilising healthcare utilisation as an outcome measure is a crude tool with significant limitations $\&$ the need to agree tailored outcome measures based on the type of intervention to assess the impact of IPMS.

\section{Reasonable adjustments for autistic adults}

Nazish Hashmi* and Conor Davidson

Leeds and York NHS foundation trust

${ }^{*}$ Corresponding author.

doi: 10.1192/bjo.2021.253

Aims. To embed the use of reasonable adjustments for adults with autism within in mental health services.

Objectives. The objectives of the project are as follows:

To identify how many service users with a diagnosis of autism are under care of local mental health services

Is there evidence that reasonable adjustments were considered for these service users

If identified as needing reasonable adjusments is there evidence of such adjustments being made

Method. We looked at service users with an established diagnosis of autism under care of Leeds and York NHS foundation trust to ascertain if reasonable adjustments have been considered. The audit is based on guidelines provided by Think Autism-department of health statutory guidance 2014. This is based on autism act 2009.

Data were collected for 30 cases in mainstream mental health services undr care of various teams including inpatient and community.

Result. It was identified that in only $2 / 30$ cases reasonable adjustments were considered and agreed upon. Only 1/30 service users had a disability status updated on electronic patient records. None of the service users had a hospital passport or reasonable adjustment care plan completed.None of the records had "good evidence" of reasonable adjustments.

These findings point to a wider issue for the trust as well as natioanlly as it indicates that autism is not being adequately taken into account for patients accessing our services. Due to the lack of reasonable adjustments adults with autism are potentially at increased risk to disengage leading to deterioration in their mental state and increase in risks.

Conclusion. These findings point to a wider issue for the trust as it indicates that autism is not being adequately taken into account for patients accessing our services. Due to the lack of reasonable adjustments adults with autism are potentially at increased risk to disengage leading to deterioration in their mental state and increase in risks.

We recommend training in autism for all healthcare professionals in the trust to improve their understanding of autism, including making reasonable adjustments.

We also recommend review trust procedure about recording diagnoses and disability status on electronic patient records. We recommend that the reasonable adjustments section on care director is more prominent and easily accessible.

We recommend that an 'autism flag' is prominent on patient records to alert staff to the presence of autism

\section{Dementia screening: an audit of screening for reversible causes of dementia}

Kim Herbert ${ }^{1 \star}$, Elspeth Richardson ${ }^{2}$, Adam Daly ${ }^{1}$

and Christine Carswell ${ }^{1}$

${ }^{1}$ NHS Lanarkshire and ${ }^{2}$ NHS Greater Glasgow and Clyde

${ }^{*}$ Corresponding author.

doi: $10.1192 /$ bjo.2021.254

Aims. This audit aimed to assess to what extent patients being referred for assessment of memory problems were receiving appropriate screening for reversible causes. We considered the blood tests recommended by the National Institute for Clinical Excellence (NICE).

Background. Research into 'reversible dementias' identified numerous common underlying causes. As a result of this NICE complied comprehensive guidance on investigations which should be performed in the initial stages of assessing patients with memory problems, ideally at a primary care level. These investigations are also crucial at the point of secondary care assessment in order to make a confident diagnosis.

Method. Details of patients referred by their GP to the Older Adult CMHT with memory problems over a one month period were collected. We then used the local laboratory database to note whether each of the eight recommended blood tests had been performed in the preceding 6 months. We measured this against an agreed standard of $95 \%$.

After the first cycle of data collection we prepared businesscard sized 'aide memoirs' for GPs that could serve as a quick reminder. These were sent out to all GPs in the area along with a letter outlining the audit findings.

Result. Overall 31 patients were included in the first cycle. 15 patients had all 8 dementia blood screens (48\%), 13 (42\%) had some of the recommended tests and 3 patients had no screening tests at all (10\%). On average patients had $76.6 \%$ of the recommended bloods completed. The most commonly completed tests were Full Blood Count (FBC) and Urea \& Electrolytes (U\&Es), with blood Glucose being the most frequently omitted.

In cycle 2, 20 patients were included. Of these patients, 10 had the full complement of screening bloods (50\%); 8 had some tests completed (40\%) and 2 patients had no screening tests complete $(10 \%)$. On average $76 \%$ of tests were completed. There was an improvement in the rate of completion of both Glucose and Liver Function Tests from cycle1.

Conclusion. This audit demonstrated that current practice does not meet the national standard in general. Our intervention produced a modest improvement in the proportion of patients who received a full complement of dementia screening tests, as well as increasing the rate of patients receiving a blood glucose as part of their screening. It would likely be beneficial to consider further intervention and a 3 rd audit cycle in due course. 